\title{
ADVANCES IN AUGMENTED REALITY (AR) FOR MEDICAL SIMULATION AND TRAINING
}

Vladimir Ivanov

Herzen State Pedagogical University of Russia

Saint-Petersburg, (Russia).

E-mail:voliva@rambler.ru ORCID: https://orcid.org/0000-0001-8194-2718

Alexander Klygach

Herzen State Pedagogical University of Russia

Saint-Petersburg, (Russia).

E-mail: voolf00@yandex.ru ORCID: https://orcid.org/0000-0002-2984-0201

Sam Shterenberg

Pavlov First Saint-Petersburg State Medical University

Saint-Petersburg, (Russia).

E-mail: sam.d.s@mail.ru ORCID: https://orcid.org/0000-0002-6428-8328

Sergey Strelkov

Herzen State Pedagogical University of Russia,

Saint-Petersburg, (Russia).

E-mail: sergin3d2d@gmail.com ORCID: https://orcid.org/0000-0002-4830-5407

Jason Levy

University of Hawaii,

Honolulu, (USA).

E-mail: jlevy@hawaii.edu ORCID: https://orcid.org/0000-0002-9978-5412

Recepción: 26/02/2020 Aceptación: 17/04/2020 Publicación: 30/04/2020

Gitación sugerida Suggested citation

Ivanov, V., Klygach, A., Shterenberg, S., Strelkov, S., y Levy, J. (2020). Advances in augmented reality (AR) for medical simulation and training. 3C Tecnología. Glosas de innovación aplicadas a la pyme. Edición Especial, Abril 2020, 303-312. http://doi.org/10.17993/3ctecno.2020.specialissue5.303-312 


\section{ABSTRACT}

Digital technologies are transforming the field of medical training, simulation and modeling. Advances in the field of virtual Augmented Reality (AR) and virtual simulation are described in detail, particularly as they relate to medical education and training. An overview of key medical simulation tools is provided in order provide foundational knowledge about this rapidly growing field. A timely and valuable original Augmented Realty system is put forward. The key components of this original system for medical training and simulation include the following three dimensions: advances in open surgery, realistic visualizations and innovative haptic was used. Each component of this Augmented Reality system is described in detail. First, the open surgery module emphasized appendectomies (the most common surgical procedures used in our model). Second, three different approaches for creating realistic and accurate 3D medical models were put forth. Third, haptic feedback involved the use of an enhanced Novint Falcon system in which a custom grip provides additional degrees of freedom. Finally, advances in game simulation, modeling and role playing are discussed for the field of emergency medicine.

\section{KEYWORDS}

Surgical Simulator, Virtual Reality, Real-time Rendering, 3D Visualization, Haptic Feedback, Open Surgery, Laparoscopy, Emergency Medicine, Simulations. 


\section{INTRODUCTION}

Medical simulations, modeling and visualizations have undergone a rapid shift the beginning of 20th century due to a number of reasons (Kron et al., 2010). First, modern approaches for less invasive surgery have redefined surgical procedures such as endoscopy and robotics surgery. Second, the dramatic rise of computing power has provided an opportunity to implement complex simulations in real-time. Finally, more accurate algorithms for rigid and soft body simulations, realistic $3 \mathrm{~d}$ visualizations, haptic controllers, and virtual reality have allowed medical simulation to be used for digital gaming rather than simply physical modeling.

Specific approaches and technologies for medical simulations for medical simulations have grown by leaps and bounds. For example, innovative research has occurred dealing with the generation of textures of irregular objects from models and photo sequences (Chen $e t$ al., 2003). The role of medical simulations has rapidly expanded throughout the healthcare field (Kunkler, 2006). This paper involves a case study of medical simulations for lap.

\section{MODERN MEDICAL SIMULATION MARKET}

\subsection{MARKET OVERVIEW}

According to Prescient \& Strategic Intelligence data, the global surgical simulation market was valued at $\$ 254.7$ million in 2017 with a growing trend. The value of this field is forecasted to increase to twice its value in 2023 (Figure 1). Another notable trend is that augmented reality (AR) and virtual reality (VR) are being used to enhance the quality and efficiency of medical training. Thus, it is expected that this market will continue to grow, and digital technologies will continue to have a major impact on the medical simulation field.

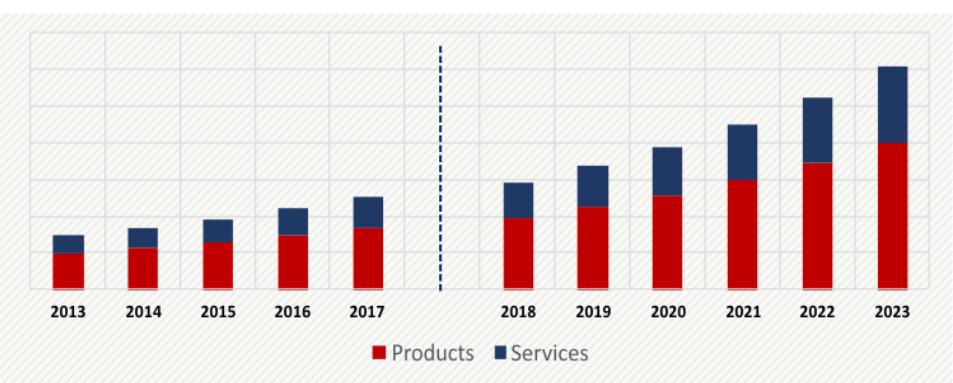

Figure 1. Worldwide surgical simulation market by offering (2013-2023). 


\subsection{MEDICAL SIMULATORS}

VirtaMed is a company primarily focused on simulator development for orthopedics, genecology and urology. The company develops surgical simulators which are designed on a single flexible plat-form with the ability to expand and add additional procedures. All simulators are combined with an anatomical model to provide the optimal tactile feedback and real-world manipulations. In addition, for better efficiency each virtual procedure allows for guided training: specific colored hints and ghost tools show trainees how to perform different tasks (Figure 2).
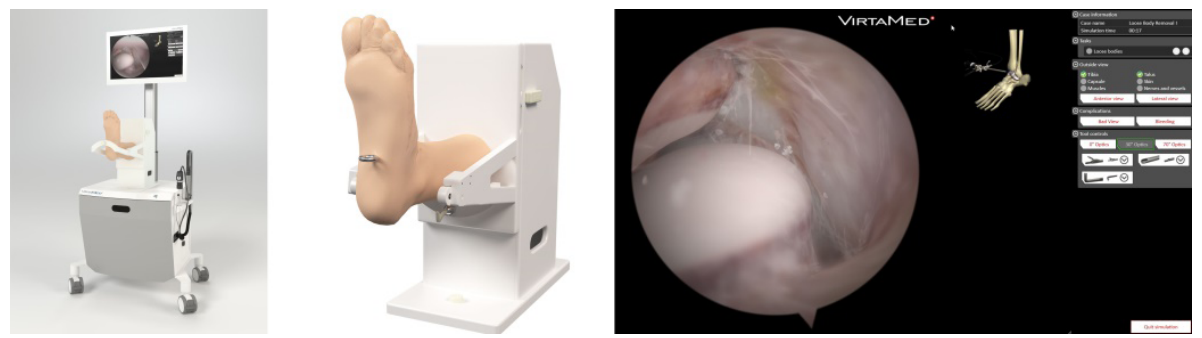

Figure 2. ArthroS Ankle by VirtaMed AG.

NeuroVR is a platform for neurological training that enables neurosurgeons to practice skills with the help of virtual reality (Figure 3). Such a system does not depend on real life models but uses haptic controllers for VR manipulations. The range of allowed exercises are derived from actual patient images, which provides more realistic and accurate images of surgical procedures. The system also captures objective metrics and measures the proficiency of procedures in order to track educational progress.
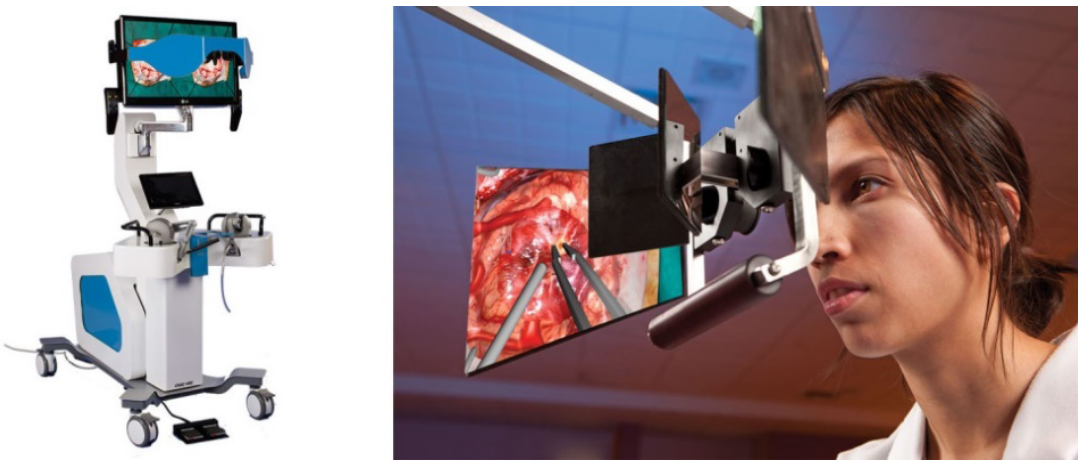

Figure 3. NeuroVR system with stereoscopic microscopic view.

SurgicalScience is a company which develops various simulation products, mostly for laparoscopy and endoscopy. The LapSim product is designed to improve psychomotor 
skills using virtual reality with haptic feedback (Figure 4). It features different modules for laparoscopic exercises that arrange from navigation to suturing. This system also has a portable version known as "LapSim essence".
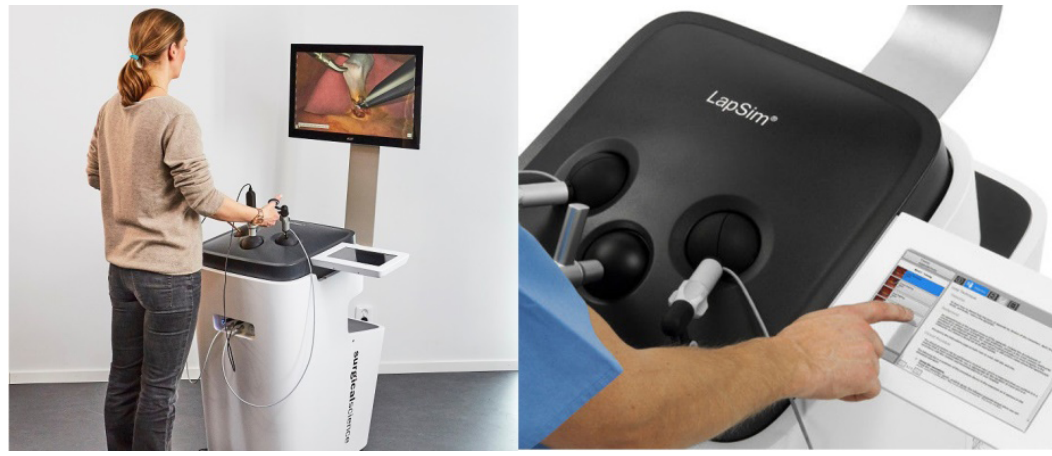

Figure 4. LapSim with in-house developed haptic system.

\section{AN ORIGINAL APPROACH TO THE DEVELOPMENT OF MEDICAL SIMULATION}

\subsection{KEY ASPECTS}

Most surgical simulators that are currently available on the market have weaknesses ranging from simplified 3D visualizations to a limited ability to perform open surgery. Most existing modeling tools are also limited because they have been designed primarily for specific surgical approaches, like endoscopy. For this reasons we develop an open surgery simulator for carried out an appendectomy with realistic visualizations using haptic feedback (Figure 5).

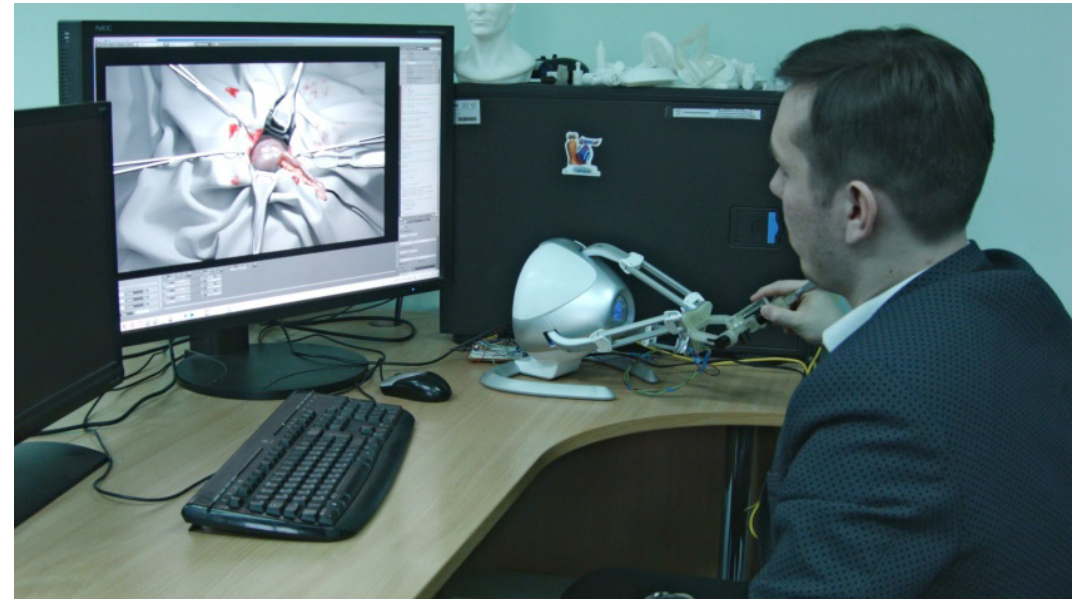

Figure 5. Surgery simulator based on open appendectomy with haptic feedback. 


\subsection{REALISTIC VISUALIZATION}

Despite advanced real-time rendering solutions that are currently available, it is still difficult to produce realistic images in a surgical simulator due to a software limitations. For example it is difficult to incorporate graphics solutions onto a complex modeling engine (that captures the physics of the system). To overcome this challenge the developed system is based on a modern game engine which allows for the use of physically based shader models and enhances it with a customized physics engine to work with soft tissue (Figure 6).

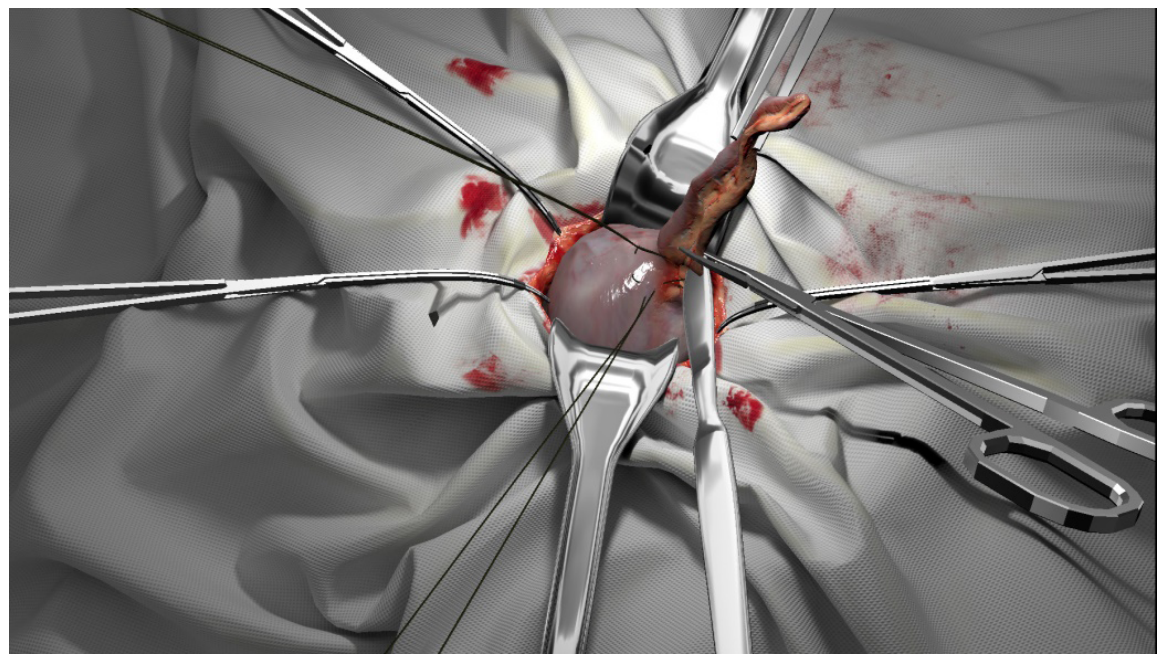

Figure 6. The stage, where the mesoappendix is dissected.

\subsection{CREATING MODELS BASED ON PATIENT' S DATA AND ANATOMICAL ATLASES}

More accurate modeling results may be obtained by a number of approaches. One method involves taking photos from an actual surgical procedure and extracting textures from these images (Figure 7). It is also possible to build models based on a sequence of photos using photogrammetry solutions.

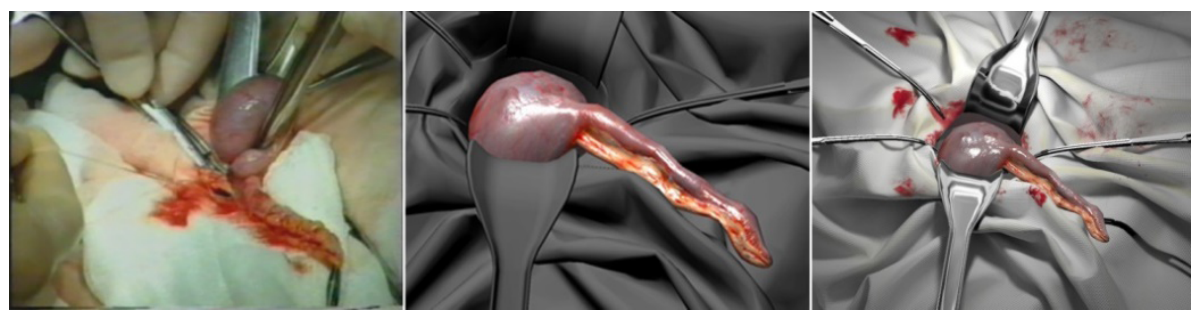

Figure 7. Photo from surgery procedure (left), appendix model with textures (center), overall view in surgical simulator (right). 
Another method involves building models based on MRI or CT data. For instance, a heart model with specific pathologies can be recreated with the help of MRI and contouring data. This is achieved through multiple stages (Figure 8). At first, we built model of ventricles from countering data and then projected a master heart model onto them and finally added textures based on real-life heart images.

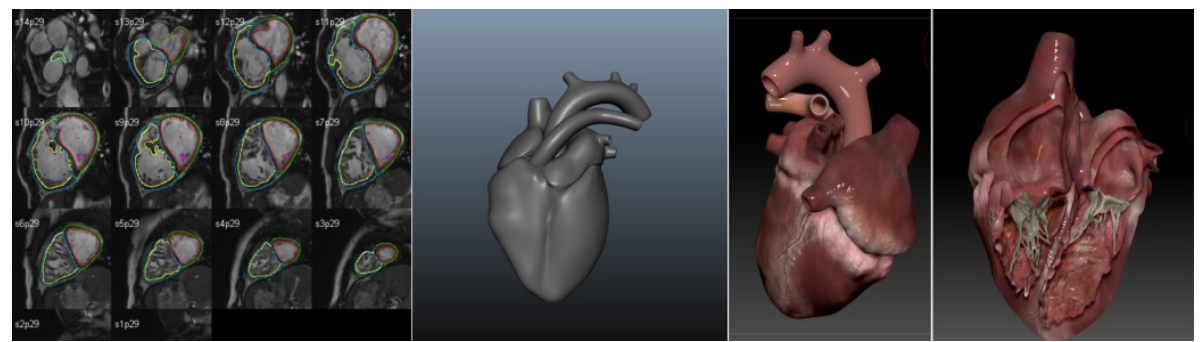

Figure 8. MRI with contouring data (left), reconstructed heart $3 \mathrm{~d}$ model (center), heart $3 \mathrm{~d}$ model with textures and internal structures (right).

In complex cases, where photography or tomography is not enough to build a full model, anatomical atlases are used: knowledge and data from multiple atlases like Gray's anatomy and $3 \mathrm{~d}$ atlas of human body were combined to reveal the position of the larynx. This helped accurately showcase the location of the larynx in comparison other anatomical structures like skull, and muscles (Figure 9).
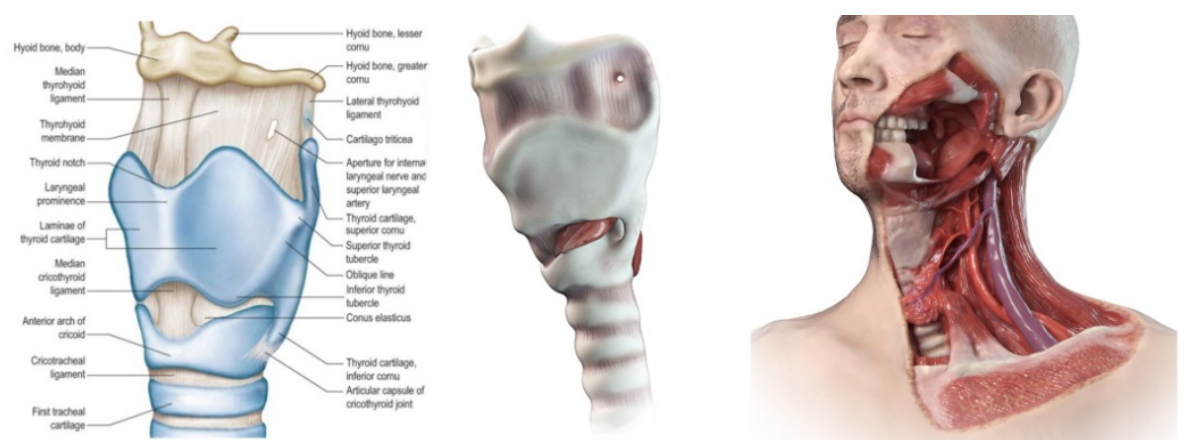

Figure 9. Larynx image from Gray's anatomy atlas (left), 3d model of larynx (center), neck section with larynx (right).

\subsection{HAPTIC FEEDBACK}

In order to achieve fully realistic visualization our system is supplemented with haptic feedback. This helps to sense virtual 3D objects and allows the development of proper psychomotor skills for surgeons. The Novint Falcon Haptic device was used as the foundation to implement this haptic feedback technology. While this tool is designed primary for games, 
it also provides accurate haptic feed-back with three degrees of freedom (DOF). This device is also the cheapest haptic system on the market, thereby reducing the overall cost of simulator significantly.

In order to use this device as a tool for surgical simulations the system was redesigned in several ways. For example a custom designed grip was developed with extra three DOF to allow for the creation of a tilting surgical tool (Figure 10). This tool is based on absolute hall encoders and transmits data as separate stream through a digital-to-analog converter. In addition, it has a slot for swapping different surgical instruments.
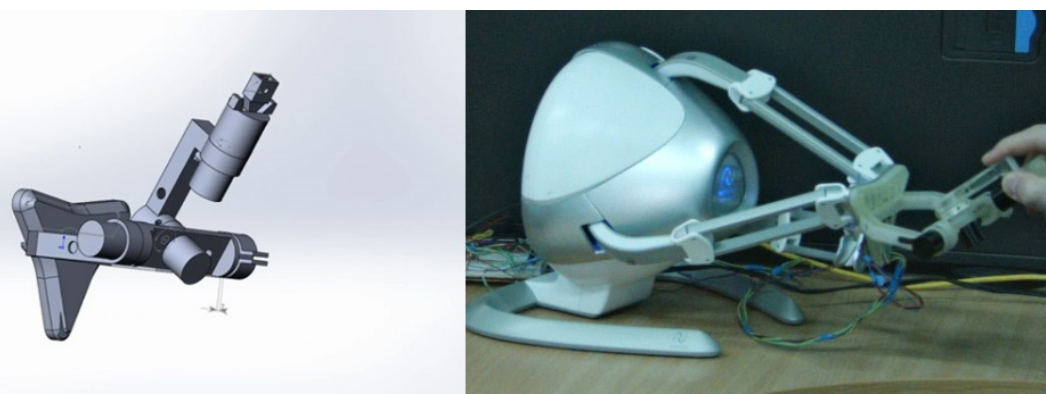

Figure 10. Custom designed grip to provide extra 3-degree of freedom.

\subsection{LAPROSCOPIC SURGICAL SIMULATORS}

There are a number of laparoscopic surgical simulators available on the market. Laparoscopic training simulators have been used effectively to enhance laparoscopic surgery training scenarios. For example, Sauerland, Jaschinski and Neugebauer (2010) examined the use of laparoscopic versus open surgery for suspected appendicitis. Our proposed Laparoscopic Surgery Simulator can demonstrate virtually all major abdominal surgical procedures and assist surgeons in learning a range of surgical methodologies. Our open surgery simulator constitutes a timely, complex and important niche simulator tool. The aforementioned solutions for creating $3 \mathrm{~d}$ models and haptic feedback can help to achieve more realistic laparoscopic surgery visualization and modeling in a cost-efficient way.

\section{GAMES, ROLE PLAYING AND SIMULATIONS FOR EMERGENCY PHYSICIANS AND THE COVID-19 PANDEMIC}

There is a need for better-trained emergency physicians in the COVID-19 era. Emergency physicians and other educational, research, and practitioners in the health field must in- 
creasingly have simulation experience to make crucial decisions within a highly politicized, volatile and pressurized context. The education of future generations of emergency physicians and emergency management professionals must draw across the boundaries of physical/social science, technology, engineering, and mathematics. The COVID-19 pandemic has shown that traditional approaches to responding to health disasters, reducing risk and mitigating losses are inadequate. Starting in the 1950s, the emphasis was on the disciplines of civil defense and humanitarian relief.

Role play, scenario methods and game simulations have been successfully used across a wide variety of learning environments ranging from military training, high school driver's education to the diplomatic art of negotiation. Role play and game simulation provide a learning-by-doing experience and thus have been shown to increase emergency management effectiveness in various application ranging from hospital disaster planning and public health to the mental and social aspects of health emergencies. Modeling and simulation tools have been shown to build higher cognitive skills for emergency management training and education and to help with individual and group learning in crisis simulations. There are a number of emergency medicine fields where role play and game simulations are used successfully in improving graduate and undergraduate learning outcomes. Despite the successes of role play and game simulations across a wide range of educational environments, there is a need for more such learning tools within in emergency healthcare education.

Our ongoing research seeks provide a bridge between the emergency response focus generally filled by community and technical colleges and theoretical focus of graduate level courses. Future work seeks to develop prototype simulations for undergraduate emergency medical curricula. A further outcome of the project will be a framework and workplan to leverage the lessons learned in this paper for complete development, implementation and faculty support materials of emergency medicine simulations.

\section{REFERENCES}

Ghen, R., Lu, D., \& Pan, Y. (2003). Generating Textures of Irregular Objects from Models and Photo Sequences. Fournal of Image and Graphics, 8. 
Kron, F. W., Gjerde, G. L., Sen, A., \& Fetters, M. D. (2010). Medical student attitudes toward video games and related new media technologies in medical education. $B M C$ Medical Education, 10(50), 1-10. https://doi.org/10.1186/1472-6920-10-50

Kunkler, K. (2006) The role of medical simulation. The International fournal of Medical Robotics and Computer Assisted Surgery, 2(3), 203-210. https://onlinelibrary.wiley.com/ doi/10.1002/rcs.101

McDermott, W. (2018). The PBRguide. Part 1. Allegorithmic. https://academy.substance3d. $\mathrm{com} /$ courses/the-pbr-guide-part-1

Sauerland, S., Jaschinski, T., \& Neugebauer, E. A. (2010). Laparoscopic versus open surgery for sus-pected appendicitis. Cochrane Database of Systematic Reviewes, (10). https://doi.org/10.1002/14651858.CD001546.pub3 